\title{
Simulation of the effect of wiggler imperfections on harmonic generation in two-beam free-electron lasers
}

\author{
M. Zahedian* and B. Maraghechi \\ Department of Physics, Amirkabir University of Technology, P.O. Box 15875-4413, Tehran, Iran
}

(Received 19 December 2011; published 1 May 2012)

\begin{abstract}
A three-dimensional simulation of a free-electron laser (FEL) with two beams is used to study the sensitivity of the third harmonic due to wiggler imperfections. In the two-beam FEL, for a fundamental wavelength of $107.5 \mathrm{~nm}$, the power will be converted to the third harmonic at a shorter wavelength, in this case in the extreme ultraviolet at $35.8 \mathrm{~nm}$. In this arrangement, the fundamental resonance of the higher energy beam coincides with the third harmonic of the lower energy beam, for this energy conversion to take place. For enhanced focusing, a planar wiggler with parabolic pole face is considered. Investigation of the effect of wiggler errors on the efficiencies of harmonic and fundamental resonance of the two-beam and the one-beam FEL shows that the average efficiency for the third harmonic in the two-beam FEL is decreased by $36 \%$ while the reduction of average efficiency for the fundamental of the two-beam is $55 \%$ and for the third harmonic of the one-beam is $48 \%$. This shows that the third harmonic radiation in the two-beam FEL is less sensitive to wiggler imperfection compared to its fundamental as well as the third harmonic in the one-beam FEL. The reason is that the energy that transfers to the third harmonic of the two-beam FEL comes from both electron beams. It was also found that, for almost all cases, standard deviation increases with an increasing level of wiggler imperfection while, for the two-beam FEL, saturation length of the fundamental resonance decreases and the third harmonic increases with increasing wiggler imperfection.
\end{abstract}

DOI: 10.1103/PhysRevSTAB.15.050702

PACS numbers: 41.60.Cr, 02.60.Cb

\section{INTRODUCTION}

There are considerable interests in free-electron lasers (FELs) to produce fully coherent, high-peak power electromagnetic radiation in the extreme ultraviolet (XUV) to hard $\mathrm{x}$ rays for many areas of science and technology. The diffraction limit of the x-ray FEL is 3 orders of magnitude lower than that of the high-power optical laser. This makes the x-ray FEL more favorable compared to the optical laser, because the laser may be focused into a much smaller size, which is of the order of $1 \mathrm{~nm}$ [1]. The most exciting property of the $\mathrm{x}$-ray FEL is its capability to image ultrasmall objects and record ultrafast events both on atomic and molecular scales.

As the power of the fundamental resonance grows, the electron beam develops a microbunching structure in the pondermotive potential formed by the beating of the wiggler and radiation fields. This microbunching, in turn, gives rise to enhanced nonlinear harmonic growth and can become the dominant harmonic growth mechanism [2-7]. The alternative method of the two-beam FEL was proposed to generate more intensified short wavelength power based on harmonic generation mechanism $[8,9]$. The multiple

\footnotetext{
*zahedian_maryam@yahoo.com
}

Published by the American Physical Society under the terms of the Creative Commons Attribution 3.0 License. Further distribution of this work must maintain attribution to the author(s) and the published article's title, journal citation, and DOI. electron beam FEL has also been studied in Ref. [10] in three dimensions but the harmonic up-conversion was not considered.

At $\mathrm{x}$-ray wavelengths, the FEL interaction is extremely sensitive to wiggler imperfections [11]. It has been shown in Ref. [11] that, since the nonlinear harmonics are driven by the growth of the fundamental, the sensitivity of the harmonics to wiggler imperfections mirrors that of the fundamentals. At longer wavelengths, simulations of wiggler imperfections are also reported [12,13]. A randomwalk model for the electron trajectories has been exploited to study the effects of random wiggler errors on spontaneous emission [14] and the linear gain $[15,16]$. In the nonlinear modeling of wiggler field errors in Refs. [16-18], an analytical model of the random walk is used in which electron trajectories are averaged over the wiggler period.

The purpose of the present study is to use the threedimensional simulation code modified MEDUSA to study the effects of wiggler imperfections on the third harmonic of the two-beam FEL, at XUV, with the wavelength of $35.8 \mathrm{~nm}$. Since we did not have access to the original MEDUSA code [6], we first rewrote the code and then modified it to study the two-beam FEL [9]. It was found that the third harmonic radiation in two-beam FEL is less sensitive to wiggler imperfection compared to its fundamental as well as the third harmonic in the one-beam FEL. In Sec. II, the problem under consideration is described. In Sec. III, simulation results for wiggler imperfection are presented. In Sec. IV, concluding remarks are made. 


\section{DESCRIPTION OF THE PROBLEM}

In the two-beam frequency up-conversion, two relativistic electron beams with different energies are used in a FEL [8,9]. The lower energy beam is chosen so that its third harmonic coincides with the fundamental resonance of the higher energy beam. It has been shown in Ref. [8] that, by seeding the fundamental resonance of the lower energy beam, this beam will be bunched at its fundamental resonance as well as at its harmonics. This will lead to a strong coupling between the two beams because the fundamental resonance of one is the same as the third harmonic of the other. Since the third harmonic is driven by both electron beams, it will be strongly amplified.

This configuration will be simulated in three dimensions using the modified MEDUSA code [9], which is based on the source dependent expansion of radiation. Since the planar wiggler is used, the Hermite-Gaussian model expansion will be employed. The field equations along with threedimensional force equations for an ensemble of electrons are integrated simultaneously. Orbit equations are not averaged over the wiggler period and modified MEDUSA treats the evolution of the electromagnetic fields selfconsistently and it can propagate the electron beam through arbitrary magnetic structures.

The simulation code modified MEDUSA was first introduced in [9] and is based on the three-dimensional code MEDUSA [6]. Because MEDUSA was not available, it was first rewritten in [9] and its validity was checked against [6]. It was subsequently modified to be used for the two-beam FEL.

The specific example consists of a two-beam FEL with the lower energy beam with an energy of $380.185 \mathrm{MeV}$, a current of $300 \mathrm{~A}$, and an initial radius of $0.02 \mathrm{~cm}$ and the higher energy beam with an energy of $799 \mathrm{MeV}$ a current of $600 \mathrm{~A}$, and an initial radius of $0.02 \mathrm{~cm}$. In the case of one-beam FEL only the slow beam will be considered. The amplitude of the wiggler field is $10.06 \mathrm{kG}$, its period is $3.3 \mathrm{~cm}$, and the length of the entry taper region is $N_{w}=10$ wiggler period. For these parameters, the wavelength of the fundamental takes place at $107.5 \mathrm{~nm}$ and the third harmonic is in XUV at $35.8 \mathrm{~nm}$. The system is studied in the absence of an energy spread while the electron beam distributions are Gaussian and the normalized emittance for both electron beams is chosen to be $3 \pi \mathrm{mm} \mathrm{mrad}$.

\section{SIMULATION RESULTS WITH WIGGLER IMPERFECTION}

We consider a single-segment parabolic pole face (PPF) wiggler so that its enhanced focusing will conveniently eliminate the need for external focusing. Although other designs for wiggler exist, the PPF wiggler will serve as a convenient wiggler model to show the sensitivity of the frequency up-conversion of the two-beam FEL to wiggler imperfections. The three-dimensional PPF wiggler may be written as [19-22]

$$
\begin{aligned}
& \mathbf{B}_{w}(\mathbf{x})= {\left[B_{w}(z)+\Delta B_{w}(z)\right]\left\{\operatorname { c o s } k _ { w } z \left[\mathbf{e}_{x} \sinh \left(\frac{k_{w} x}{\sqrt{2}}\right)\right.\right.} \\
&\left.\times \sinh \left(\frac{k_{w} y}{\sqrt{2}}\right)+\mathbf{e}_{y} \cosh \left(\frac{k_{w} x}{\sqrt{2}}\right) \cosh \left(\frac{k_{w} y}{\sqrt{2}}\right)\right] \\
&\left.-\sqrt{2} \mathbf{e}_{z} \cosh \left(\frac{k_{w} x}{\sqrt{2}}\right) \sinh \left(\frac{k_{w} y}{\sqrt{2}}\right) \sin k_{w} z\right\} \\
& B_{w}(z)= \begin{cases}B_{w} \sin ^{2}\left(\frac{k_{w} z}{4 N_{w}}\right) 0 & \leq z \leq N_{w} \lambda_{w} \\
B_{w} & N_{w} \lambda_{w}<z\end{cases}
\end{aligned}
$$

where $k_{w}=2 \pi / \lambda_{w}$ is the wiggler wave number and $\Delta B_{w}$ is the random variation in amplitude representing the wiggler error. $B_{w}(z)$ in Eq. (2) is the systematic (nonrandom) variations in the amplitude and essentially describes the injection of the electrons by an adiabatic entry taper over the first $N_{w}$ wiggler periods and a constant wiggler magnetic field afterwards.

The total length of the wiggler is divided into regular intervals. The random component of amplitude is chosen at points connecting these intervals using a random number generator and two neighboring points are connecting by a continuous curve. The interval between the random fluctuations in the amplitude is assumed to be $\Delta z=\lambda_{w} / 2$, which assumes two pole faces per wiggler period. Therefore, a random series of amplitudes $\left\{\Delta B_{n}\right\}$ is generated, with $\Delta B_{n} \equiv \Delta B_{w}(z=n \Delta z)$. The entry taper region has been chosen to be free from fluctuations with $\Delta B_{n}=0$ for $0 \leq n \leq 1+2 N_{w}$ to make sure that amplitude is always positive. $\Delta B_{w}(z)$ is assumed to vary between these points by

$$
\Delta B_{w}(n \Delta z+\delta z)=\Delta B_{n}+\left[\Delta B_{n+1}-\Delta B_{n}\right] \times \sin ^{2}\left(\frac{\pi}{2} \frac{\delta z}{\Delta z}\right)
$$

where $0 \leq \delta z \leq \Delta z$. It needs to be mentioned that this formulation can model the effects of pole-to-pole variations in any particular wiggler magnet.

Before analyzing the effects of the wiggler imperfections, it is useful to study the performance of the two-beam FEL with an ideal wiggler $\Delta B_{w}=0$. Figure 1 shows the evolution of the power of the fundamental resonance and harmonic up-conversion at the third harmonic of the twobeam FEL with the distance along the wiggler. Contrary to the nonlinear harmonic generation by a single beam, the intensity of the third harmonic with shorter wavelength is larger than that of the fundamental wavelength. This shows that, when the fundamental resonance of the lower energy beam is seeded, the larger portion of energy is upconverted to the third harmonic to obtain higher power. This is because the third harmonic is driven by both beams and it consists of the superposition of two different kinds of amplifying radiation; one being the fundamental resonance 


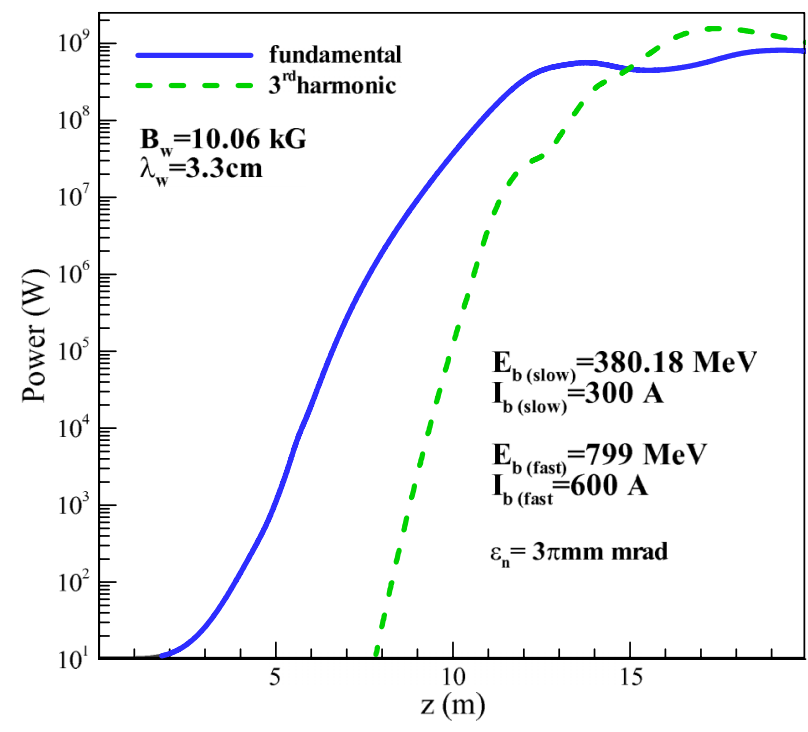

FIG. 1. Evolution of power of the fundamental and third harmonic for an ideal wiggler of the two-beam FEL.

of the high energy beam and the other the third harmonic of the low energy beam.

To study the wiggler imperfection effects, an rms fluctuation level will be chosen and several fluctuating distributions will be generated using a random number generator. A complete analysis requires a large number of simulation runs with different random wiggler fluctuations to obtain statistically reliable results. We will use 30 different randomly generated fluctuation distributions to obtain a good statistics for the ensemble average of the saturated power.

Random number generator in FORTRAN produces random numbers between 0 and 1 with uniform distribution and with constant mean value and standard deviation of $1 / 2$ and $1 / \sqrt{12}$, respectively [23]. To generate a set of random numbers $\left\{\Delta B_{n}\right\}$ with optional mean value and rms, we need to introduce rescaling $y=c x+d$, where $x$ and $y$ are the old and new set of random numbers respectively, and $c$ and $d$ are constants. By determining $c$ and $d$ for given rms and mean value, the new set is obtained. It is most natural to consider a random fluctuation which is relatively uniform over the interaction region (i.e., $\left\langle\Delta B_{w}\right\rangle=0$ ) [12]. The mean value of the modified distribution is $\mu_{y}=d+$ $c \mu_{x}$ [23], where $\mu_{x}$ is the mean value of the old distribution. For $\mu_{y}=d+c \mu_{x}=0$, a relation between $c$ and $d$ is obtained as $c=-d / \mu_{x}$. Then we need to find standard deviation as there is a relation between rms and standard deviation. By doing some calculations, standard deviation of the new set can be obtained as $\sigma_{y}=c \sqrt{1 / 12}$ [23]. Definitions of root mean square and standard deviation are $\mathrm{rms}=\sqrt{\sum_{i} y_{i}^{2} / n}$ and $\sigma_{y}=\sqrt{\sum_{i}\left(y_{i}-\mu_{y}\right)^{2} / n}$, where $n$ is the number of poles along the wiggler length. Therefore for $\mu_{y}=0, \mathrm{rms}=\sigma_{y}, c=\sqrt{12} \mathrm{rms}$, and $d=$ $-\sqrt{3}$ rms. Thus, by determining $c$ and $d$ and generating

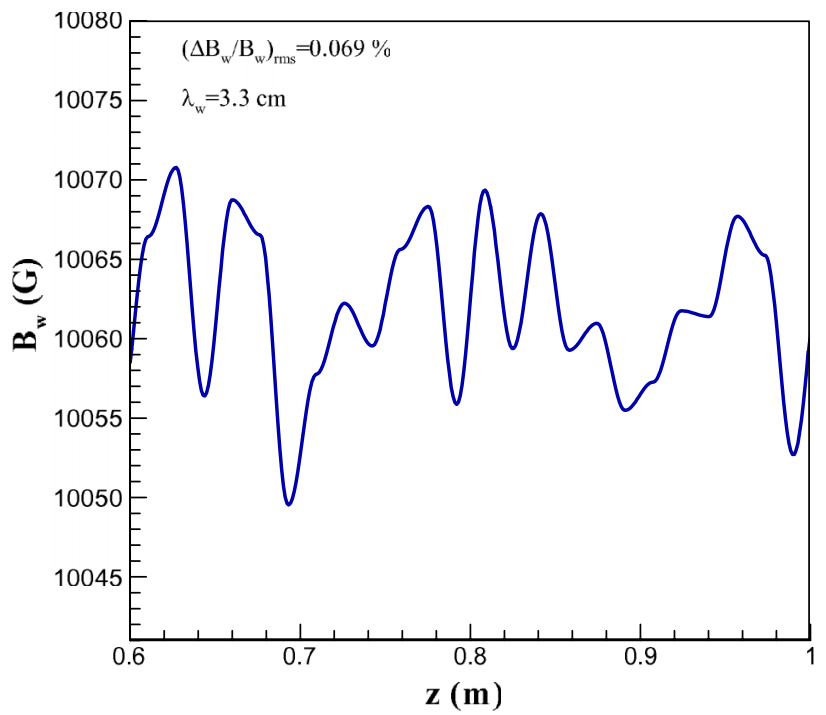

FIG. 2. Fluctuations in the wiggler field due to imperfections.

random numbers by a random number generator, a distribution with a given rms is obtained.

Fluctuation of the wiggler field from pole to pole is shown in Fig. 2 for $\left(\Delta B_{w} / B_{w}\right)_{\mathrm{rms}}=0.069 \%$.

(a)

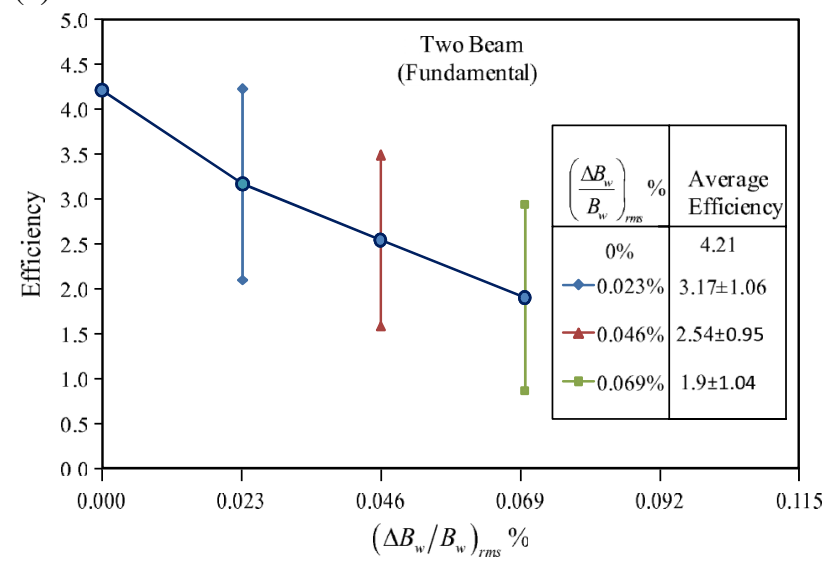

(b)

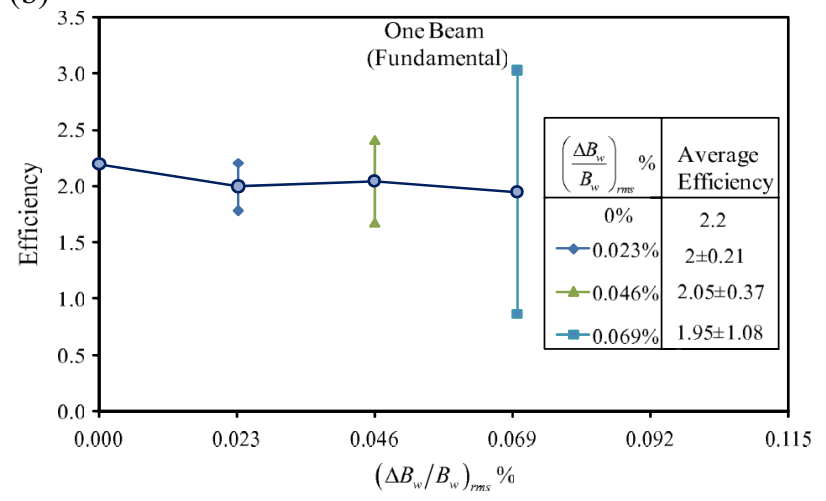

FIG. 3. Variation in efficiency at the fundamental for the (a) two-beam FEL and (b) one-beam FEL. 
Variation of the average efficiency (based on the peak power at saturation) with the rms fluctuation level of the wiggler imperfections $\left(\Delta B_{w} / B_{w}\right)_{\mathrm{rms}}$ is shown in Figs. 3 and 4 , where dots represent the average efficiency and error bars denote the standard deviation. Figure 3 is for the fundamental frequency and Fig. 4 is for the third harmonic. Plot (a) is for the two-beam and plot (b) is for the one-beam FEL. It can be seen that in general, except for a couple of cases, average efficiency decreases with increasing $\left(\Delta B_{w} / B_{w}\right)_{\text {rms. }}$. However, almost for all cases, standard deviation increases with increasing level of wiggler imperfection. When $\left(\Delta B_{w} / B_{w}\right)_{\mathrm{rms}}$ is increased to $0.069 \%$, the average efficiency for the fundamental is decreased by $55 \%$ and $11 \%$ for the two-beam and the one-beam (slow beam) FEL, respectively, while for the third harmonic, this drop is by $36 \%$ and $48 \%$. The third harmonic in the twobeam FEL in Fig. 4(a), which is of the main concern herein, tolerates $0.046 \%$ of rms imperfection of the wiggler. In calculating the efficiency of the third harmonic in the two-beam FEL, the input energy is taken as the sum of the kinetic energy of both electron beams; however, only the energy of the slow beam is used for calculating the

(a)

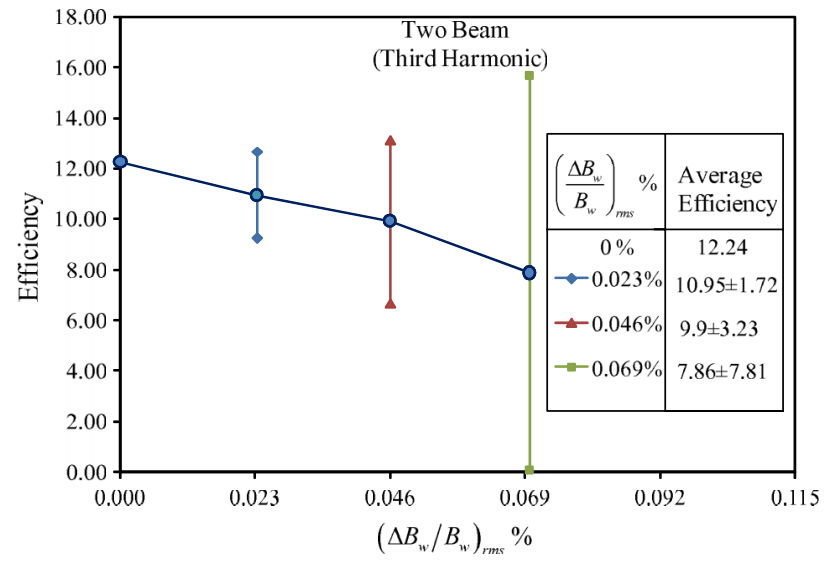

(b)

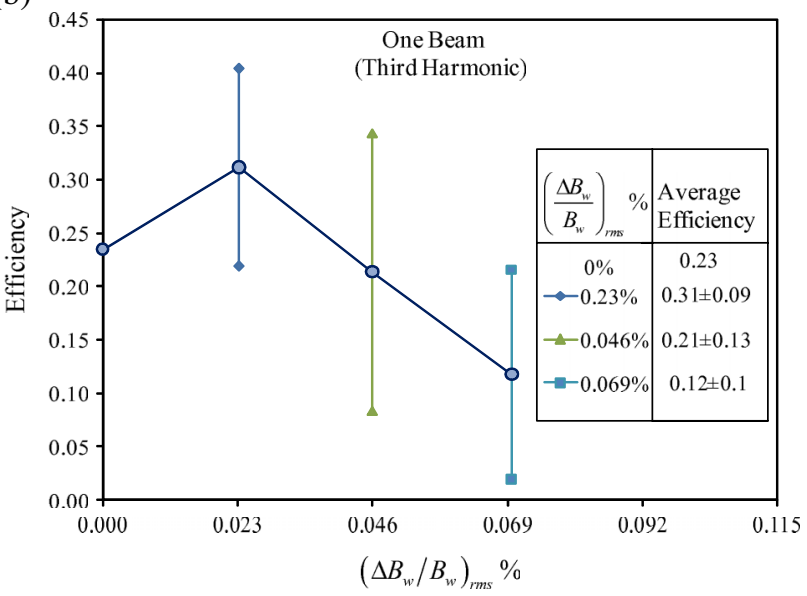

FIG. 4. Variation in efficiency at the third harmonic for the (a) two-beam FEL and (b) one-beam FEL. efficiency of the fundamental, so it can be compared with that of the one-beam FEL. It can also be observed that the largest efficiency drop belongs to the fundamental of the two-beam FEL while its third harmonic is relatively less sensitive to the wiggler imperfection. The saturation efficiencies are calculated with the peak power at saturation.

It can be seen in Fig. 3 that the fundamental for the onebeam FEL is much less sensitive to $\left(\Delta B_{w} / B_{w}\right)_{\mathrm{rms}}$ as compared to the one for the two-beam FEL and its efficiency drops much less. The reason is that in the two-beam FEL much more energy goes into the third harmonic compared to the one-beam case. The fact that the variation of average saturation efficiency for the third harmonic is not as regular as that at the fundamental for the one-beam FEL is consistent with Ref. [11] for shorter wavelength. For the twobeam, however, the general behavior of the variation of efficiency with $\left(\Delta B_{w} / B_{w}\right)_{\text {rms }}$ for the fundamental and the third harmonic is much more similar. This is because unlike the one-beam case, the saturated power levels for both wavelengths are almost of the same order.

Figure 5 shows the variation of saturation length for different rms fluctuations, for fundamental and third

(a)

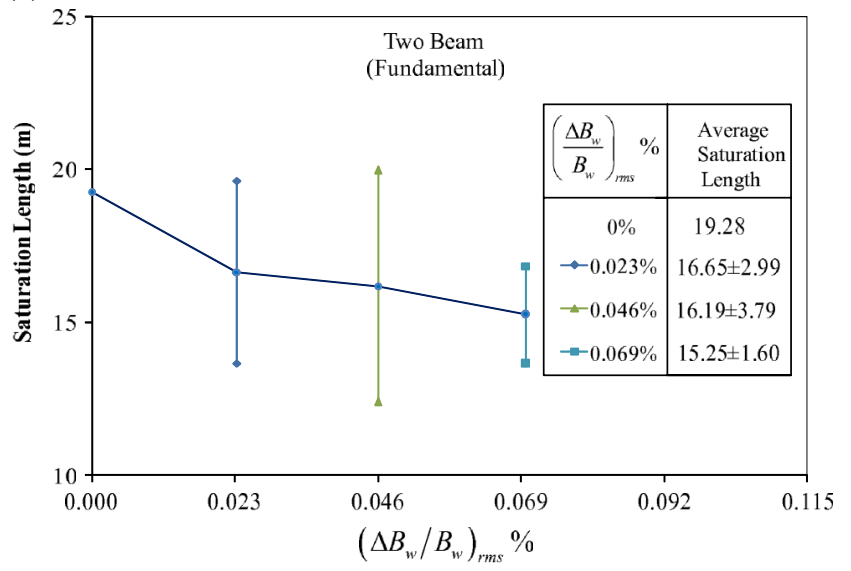

(b)

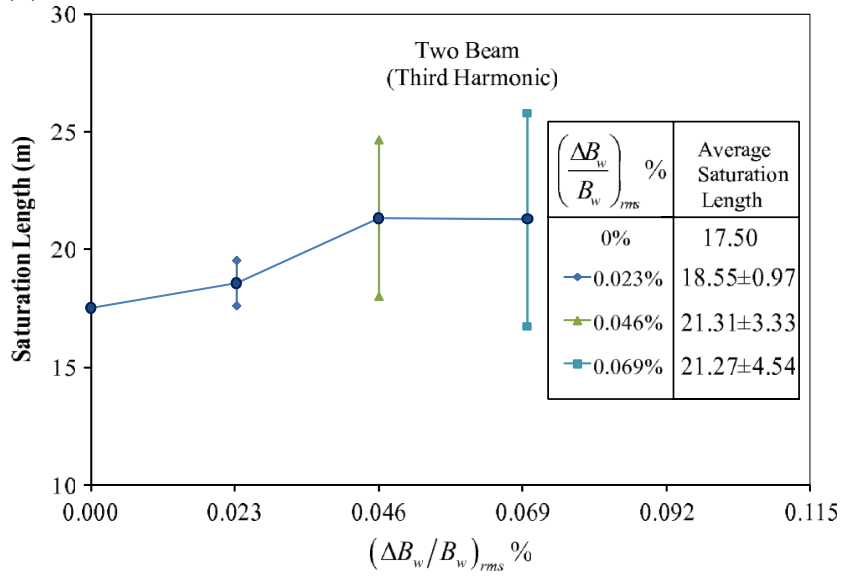

FIG. 5. Variation of saturation length of the fundamental and third harmonic of the two-beam FEL. 
harmonic of the two-beam FEL. It shows that by increasing the level of imperfection, the saturation length of fundamental radiation decreases while the saturation length of the third harmonic increases and for almost both cases the standard deviation of the saturation lengths increases with an increasing level of error.

Comparison of the results of the present study with other works on investigating the effect of wiggler imperfection on saturated powers [11-13] shows that, for shorter wavelengths, the FEL performance becomes more sensitive to wiggler errors. In Ref. [11], for wavelengths in the Angstrom region, the variations about the mean in the magnetizations of the individual magnets comprising the wiggler tolerates $0.015 \%$ of rms imperfection of the wiggler [11] while in our study, in the $35.8 \mathrm{~nm}$ region, its effect is ignorable if the error is less than $0.046 \%$ and in microwave regions $[12,13], 5 \%$ error is still benign and does not have a noticeable effect on efficiency.

\section{CONCLUSION}

A three-dimensional and nonlinear simulation MEDUSA code is used to study the effect of wiggler imperfection on harmonic up-conversion in a two-beam FEL. The results demonstrate that the average efficiency of the third harmonic in the two-beam FEL decreases $36 \%$ while the reduction of average efficiency for the fundamental of the two-beam is $55 \%$ and for the third harmonic of the one-beam is $48 \%$. Therefore, the third harmonic of the two-beam FEL is somewhat less sensitive to the wiggler imperfection compared to its fundamental as well as to the third harmonic of the one-beam FEL, because it is fed by both beams. This mode was however found to be much more sensitive compared to the fundamental of the onebeam FEL (the average efficiency drop for the third harmonic of the two-beam is $36 \%$ while the drop for the fundamental of the one-beam is $11 \%$ ) as it is always the characteristic of nonlinear harmonics.

[1] S. Krishnagopal, V. Kumar, S. Maiti, S. S. Prabhu, and S. K. Sarkar, Curr. Sci. 87, 1066 (2004) [http://www.iisc .ernet.in/currsci/oct252004/1066.pdf ].

[2] R. Bonifacio, L. De Salvo, and P. Pierini, Nucl. Instrum. Methods Phys. Res., Sect. A 293, 627 (1990).

[3] W. M. Fawley, H.-D. Nuhn, R. Bonifacio, and E.T. Scharlemann, Proceedings of the Particle Accelerator
Conference 1995, Dallas, Texas (IEEE, New York, 1995), Vol. 5, p. 219.

[4] S. G. Biedron, R. Bartolini, F. Ciocci, G. Dattoli, W. M. Fawley, G. Felici, H.P. Freund, H.-D. Nuhn, P.L. Ottaviani, and A. Renieri, in LASE 2002, High-Power Lasers and Applications, Directed Energy, Laser and Beam Control Technologies, Santanu Basu, Boeing Co., Rancho Palos Verdes, CA, SPIE Proceedings Vol. 4632 (SPIE, Bellingham, WA, 2002), p. 50.

[5] G. Dattoli, L. Gianessi, P. L. Ottaviani, H. P. Freund, S. G. Biedron, and S. V. Milton, Nucl. Instrum. Methods Phys. Res., Sect. A 495, 48 (2002).

[6] H.P. Freund, S. G. Biedron, and S. V. Milton, IEEE J. Quantum Electron. 36, 275 (2000).

[7] S. G. Biedron, H. P. Freund, S. V. Milton, L.-H. Yu, and X. J. Wang, Nucl. Instrum. Methods Phys. Res., Sect. A 475, 118 (2001).

[8] B. W. J. McNeil, G. R. M. Robb, and M. W. Poole, Phys. Rev. E 70, 035501(R) (2004).

[9] M. H. Rouhani and B. Maraghechi, Phys. Rev. ST Accel. Beams 13, 080706 (2010).

[10] H. P. Freund, D. Douglas, and P. G. O'Shea, Nucl. Instrum. Methods Phys. Res., Sect. A 507, 373 (2003).

[11] H. P. Freund, S. G. Biedron, S. V. Milton, and H. D. Nuhn, IEEE J. Quantum Electron. 37, 790 (2001).

[12] H. P. Freund and R. H. Jackson, Phys. Rev. A 45, 7488 (1992).

[13] H. P. Freund and R. H. Jackson, Nucl. Instrum. Methods Phys. Res., Sect. A 341, 225 (1994).

[14] B. M. Kincaid, J. Opt. Soc. Am. B 2, 1294 (1985).

[15] W. P. Marable, E. Esarey, and C. M. Tang, Phys. Rev. A 42, 3006 (1990).

[16] L.H. Yu, S. Krinsky, R. L. Gluckstern, and J. B. J. van Zeijts, Phys. Rev. A 45, 1163 (1992).

[17] H.D. Shay and E. T. Scharlemann, Nucl. Instrum. Methods Phys. Res., Sect. A 272, 601 (1988).

[18] W.P. Marable, C. M. Tang, and E. Esarey, IEEE J. Quantum Electron. 27, 2693 (1991).

[19] H. P. Freund and J.M. Antonsen, Principle of Free Electron Laser (Chapman and Hall, London, 1996), Chap. 1.

[20] R. M. Phillips, Nucl. Instrum. Methods Phys. Res., Sect. A 272, 1 (1988).

[21] E. T. Scharlemann, J. Appl. Phys. 58, 2154 (1985).

[22] R. P. Walker, Nucl. Instrum. Methods Phys. Res., Sect. A 237, 366 (1985).

[23] W. H. Press, S. A. Teukolsky, W. T. Vetterling, and B.P. Flannery, Numerical Recipes in Fortran 77 (Press Syndicate of the University of Cambridge, New York, 1992), 2nd ed. 